\title{
Productivity, Profitability and Soil Health of Pigeonpea as Influenced by Phosphorus Levels and Bioinoculants under Eastern Uttar Pradesh
}

\author{
Amitesh Kumar Singh ${ }^{1}$, R.S. Singh ${ }^{2}$, S.P. Singh ${ }^{2}$, Narendra Kumawat ${ }^{3 *}$ and \\ Rakesh Kumar
}

\author{
${ }^{1}$ Department of Agronomy, IAS, RGSC, BHU, Barkachha, Mirzapur-231 001 (UP) India \\ ${ }^{2}$ Department of Agronomy, IAS, BHU, Varanasi-221 005 (UP) India \\ ${ }^{3}$ AICRP Maize, Zonal Agricultural Research Station, Jhabua-457661 (MP) India \\ ${ }^{4}$ ICAR-Research Complex for Eastern Region, Patna-797 106, Bihar, India \\ *Corresponding author
}

A B S T R A C T

\begin{tabular}{l} 
Ke y w o r d s \\
Bioinoculants, \\
Correlation \\
coefficient, \\
Phosphorus, \\
Pigeonpea, Quality, \\
Soil health, Yield. \\
\hline Article Info \\
\hline $\begin{array}{l}\text { Accepted: } \\
\text { 23 May } 2017 \\
\text { Available Online: } \\
\text { 10 June } 2017\end{array}$ \\
\hline
\end{tabular}

Keywords

Field experiments were carried out during kharif season of 2008-09 and 2009-10 at Agricultural Research Farm, Institute of Agricultural Sciences, Banaras Hindu University, Varanasi to study the effect of phosphorus levels and bioinoculants on pigeonpea. Results reveal that application of $75 \mathrm{~kg} \mathrm{P}_{2} \mathrm{O}_{5} /$ ha recorded the highest soil microorganisms, growth characters, seed yield (2265 and $2124 \mathrm{~kg} / \mathrm{ha}$ ), net return, B: C ratio, production and economic efficiency, being significantly superior over $25 \mathrm{~kg} \mathrm{P}_{2} \mathrm{O}_{5} /$ ha and control during both the years. During both the years of study dual seed inoculation with PSB + PGPR produced the maximum nodulation, seed yield (2314 and $2173 \mathrm{~kg} / \mathrm{ha}$ ), net return, B:C ratio, production and economic efficiency, being significantly superior to alone seed inoculation of PGPR, PSB and control. Further results showed that maximum soil nutrient $\left(\mathrm{N}, \mathrm{P}, \mathrm{K}\right.$ and $\mathrm{S}$ ) was recorded in the plot where $70 \mathrm{~kg} \mathrm{P}_{2} \mathrm{O}_{5} /$ ha applied and this treatment was found significantly superior over $25 \mathrm{~kg} \mathrm{P}_{2} \mathrm{O}_{5} /$ ha and control. Dual seed inoculation of PSB + PGPR was recorded higher organic carbon and available soil nutrients, which was significantly superior over alone seed inoculation of PGPR, PSB and control.

\section{Introduction}

Pulses are major source of protein in the diets of vegetarian peoples in the developing countries in general and particular in India. Even though India is the largest producer, consumer, processor and importer of pulses in the world, the average productivity is very low and the production is not sufficient to meet the daily requirement of the peoples (Kumawat et al., 2012). Pulses are also an excellent feed and fodder for livestock. India is the shared $24 \%$ in the global production of pulses. The major pulses are chickpea (48\%), pigeonpea $(15 \%)$, mungbean (7\%), urdbean (7\%), lentil (5\%) and fieldpea (5\%). Among the pulses pigeonpea is the fifth prominent grain legume crop in the world and occupies the second position in India after chickpea (Kumawat et al., 2013). The major pulses producing states are Madhya Pradesh, Maharashtra, Rajasthan, Uttar Pradesh, Karnataka and Andhra Pradesh, which together account for about $80 \%$ of the total production. India has the largest acreage under pigeonpea 2.66 million hectare with a 
total production of 2.47 million tonnes, respectively (Ali and Gupta, 2012). The low yield of this crop is not only due to its grown in marginal and sub-marginal land, but also inadequate and imbalanced fertilization as well as continuous use of chemical fertilizers which decrease productivity, sustainability and finally affected soil health. Among the macronutrients phosphorus is major plant nutrient which is referred to as the key element in crop production. It affects seed germination, cell division, flowering, fruiting, synthesis of fat, starch and in fact mostly all bio-chemical activities. It also induces root proliferation and nodulation. $\mathrm{P}$ has novel function of special importance in the process of energy storage and transfer. Response of phosphorus in pigeonpea has been reported by many researchers (Kumar and Kushwaha, 2006; Singh and Yadav, 2008; Kumawat et al., 2015).

Phosphorus solubilizing rhizobacteria (PSB) are capable to convert insoluble phosphorus into soluble forms, therefore used to increase the solubility of reprecipitated soil phosphorus for crop production PSB help in the solubilization of native phosphorus from rock phosphate and other readily in soluble forms of soil phosphorus by producing organic acid like citric, gluconic, succinic, lactic, oxalic, glycolic, malic, formic, maleic and $\alpha$-ketogluconic. PSB can solublize almost 15-20 kg $\mathrm{P}_{2} \mathrm{O}_{5} /$ ha per season (Chandra and Kumar, 2005 and Kumawat et al., 2010a). Positive response of PSM in pulse crops has been reported by many researchers (Kumawat et al., 2009abc; Kumawat et al., 2010b) Plant growth promoting rhizobacteria (PGPR) are a group of free-living rhizobacteria that colonies of rhizosphere and benefited to near growing roots of plants. Microorganisms of various genera were isolated as plant growth promoting rhizobacteria of which Bacillus and Pseudomonas sp. are predominant. PGPR exert a direct effect on plant growth by production of phytohormones, solubilization of inorganic phosphates, PGPR reduce the populations of root pathogens and other deleterious microorganisms in the rhizosphere, thus benefiting the plant growth reported by Podile and Kishore (2006) and Singh and Singh (2012a). Keeping this in view a field experiment was planned to enhance the yield of pigeonpea and soil health with the bioinoculants and phosphorus.

\section{Materials and Methods}

The field experiment was conducted at the Agriculture Research Farm of Department of Agronomy, Institute of Agricultural Sciences, Banaras Hindu University, Varanasi, during the kharif seasons of 2008-09 and 2009-10. The Farm is located at $25^{\circ} 18^{\prime}$ north latitude and $83^{\circ} 03^{\prime}$ east longitude with an elevation of $129 \mathrm{~m}$ above mean sea level. The experimental soil was sandy clay loam in texture having $\mathrm{pH}(7.46,7.53)$. It was moderately fertile being low in organic carbon (0.34 and $0.37 \%)$. The available N, P, $\mathrm{K}$, S was $188.7,18.5,205.3,19.3 \mathrm{~kg} / \mathrm{ha}$ in first year and 194.2, 22.4, 210.6, $21.5 \mathrm{~kg} / \mathrm{ha}$ in second year, respectively. The experiment was laid out at the same site and same plots during both the years in split plot design replicated thrice keeping phosphorus levels (control, 25, 50 and $75 \mathrm{~kg} / \mathrm{ha}$ ) in main plots and bioinoculants (control, PSB, PGPR and $\mathrm{PSB}+\mathrm{PGPR}$ ) in sub plots. Phosphorus was applied as per treatment through diamonium phosphate (DAP). A uniform dose of $\mathrm{N}, \mathrm{K}_{2} \mathrm{O}$ and sulphur@30,40 and 30 kg/ha were applied though urea, muraite of potash and elemental sulphur (90\%). Seed of crop was inoculated with phosphate solubilizing bacteria (Bacillus polymyxa), PGPR (Rhizobium + Pseudomonas florencenses) and dual inoculation with PSB+PGPR (Bacillus polymyxa + Rhizobium + Pseudomonas fluorescens) before sowing of crop. Long duration pigeonpea $c v$. Bahar was sown by 
ridge-furrow method of planting on 14 and 20 July in 2008 and 2009 and the respective crop was harvested on 06 and 12 April in 2009 and 2010, respectively. The required plant population $(60 \times 20 \mathrm{~cm})$ was obtained after a month of seeding by thinning of the plants. During first year more favorable weather conditions specially rainfall which was comparatively higher $(528.60 \mathrm{~mm})$ than second year $(420.41 \mathrm{~mm})$. During second year at flowering time (January to February) flower dropping due to frost and resulted affect yield of pigeonpea. Production efficiency and economic efficiency was calculated as following formula suggested by Kumawat et al., (2015).

\section{Results and Discussion}

\section{Effect of phosphorus}

Phosphorus levels has significant effect on microbial populations, chlorophyll content, effective nodules/plant, nodules dry weight $(\mathrm{mg})$, leaf area index, growth crop rate and plant dry weight at 50 and 100 DAS during both the years (Tables 1 and 2). Data indicated that application of phosphorus @ 75 $\mathrm{kg} / \mathrm{ha}$ recorded the highest microbial population (1.5 and $1.9 \mathrm{cfu} / \mathrm{g}$ of soil), effective nodules/plant (9.93 and 9.16) and nodules dry weight (74.41 and 69.35 $\mathrm{mg} /$ plant) and its being at par with $50 \mathrm{~kg}$ $\mathrm{P}_{2} \mathrm{O}_{5} / \mathrm{ha}$ and significantly superior over $25 \mathrm{~kg}$ $\mathrm{P}_{2} \mathrm{O}_{5} /$ ha and control, respectively. Further data showed that highest chlorophyll content (35.50 and 33.75 SPAD), leaf area index (1.57 and 1.25), crop growth rate $(0.429$ and $0.353 \mathrm{~g} /$ day/plant) and dry matter production (21.43 and 17.64) were noted with the application of phosphors@ $@ 75$ kg/ha which was significantly superior to control and 25 $\mathrm{kg} \mathrm{P}_{2} \mathrm{O}_{5} / \mathrm{ha}$ but at par with $50 \mathrm{~kg} \mathrm{P}_{2} \mathrm{O}_{5} / \mathrm{ha}$ during both the years of experimentation. Similarly, higher yield attributes viz. pods/plant, seeds/pod, 1000-seed weight
(Figs. 1, 2 and 3) and seed yield (2265 and $2124 \mathrm{~kg} / \mathrm{ha}$ ) was found with the application of $75 \mathrm{~kg} \mathrm{P}_{2} \mathrm{O}_{5} /$ ha, being significantly superior to $25 \mathrm{~kg} \mathrm{P}_{2} \mathrm{O}_{5} / \mathrm{ha}$ and control. This might be due to the fact that plants absorbed plant nutrients relatively in higher quantity because the pool of available phosphorus was already increased doses of applied phosphorus resulting enhancement of growth and yield attributes of crop. The present results agree with those of Singh and Singh (2012a) and Singh and Singh (2012b). The higher yields may be ascribed to the effect of $\mathrm{P}$ on root development, energy transformation and metabolic processes of the plant, which in term resulted in greater translocation of photosynthates towards the sink development. These results are in conformity with Singh and Ahlawat (2007) in pigeonpea, Kumawat et al., (2010b) in greengram and Singh and Singh (2012b).

Maximum net return $\left(\times 10^{3} \quad 93.43\right.$ and $87.66 /$ ha), benefit: cost ratio (3.19 and 2.99) production efficiency $(8.5$ and $7.9 \mathrm{~kg} / \mathrm{ha} /$ day $)$ and economic efficiency (349.9 and 328.3 //ha/day) was obtained with $75 \mathrm{~kg} \mathrm{P}_{2} \mathrm{O}_{5} /$ ha and this treatment, being significantly superior over $25 \mathrm{~kg} \mathrm{P}_{2} \mathrm{O}_{5} / \mathrm{ha}$ and control (Table 3). Similar findings were also made by Singh and Singh (2012b) and Ahirwar et al., (2016). Further table 3 indicated that protein content was maximum with the application of $75 \mathrm{~kg} \mathrm{P}_{2} \mathrm{O}_{5} /$ ha and lowest in control. Similarly highest protein yield was recorded with the highest level of phosphorus i.e. of $75 \mathrm{~kg}$ $\mathrm{P}_{2} \mathrm{O}_{5}$ /ha which was statistically similar to 50 $\mathrm{kg} \mathrm{P}_{2} \mathrm{O}_{5} / \mathrm{ha}$ and significantly superior to $25 \mathrm{~kg}$ $\mathrm{P}_{2} \mathrm{O}_{5} /$ ha and control. This could be ascribed due to protein content is essentially the manifestation of $\mathrm{N}$ concentration in seed. Hence, increased $\mathrm{N}$ concentration might have increased the protein content and finally protein yield. These results corroborated with the finding of Jain et al., (2007) and Singh and Singh (2012b). The maximum available soil organic carbon was recorded under $75 \mathrm{~kg}$ 
$\mathrm{P}_{2} \mathrm{O}_{5} /$ ha and this treatment has significantly superior to $25 \mathrm{~kg} \mathrm{P}_{2} \mathrm{O}_{5} / \mathrm{ha}$ and control (Table 5). Similarly, application of $75 \mathrm{~kg}_{2} \mathrm{O}_{5} / \mathrm{ha}$ recorded maximum available soil $\mathrm{N}, \mathrm{P}, \mathrm{K}$ and $\mathrm{S}$ but it was statistically at par with $50 \mathrm{~kg}$ $\mathrm{P}_{2} \mathrm{O}_{5} / \mathrm{ha}$ and significantly superior to $25 \mathrm{~kg}$ $\mathrm{P}_{2} \mathrm{O}_{5} /$ ha and control (Table 5).

It might be due to the beneficial effect of phosphorus on root growth and development and nodulation of mungbean which led to more $\mathrm{N}_{2}$-fixation and thus after harvest of crop $\mathrm{N}$ depletion under phosphorus treatment was low as compared to control. The increase in available nutrients in soil with application of phosphorus could be due to utilization of native phosphorus with increasing levels of phosphorus which resulted in building up of higher soil nutrients status. Similar findings were also made by Ahirwar et al., (2016).

\section{Effect of bioinoculants}

Among the bioinoculants, dual inoculation with PSB + PGPR was significantly recorded higher microbial population $(1.5$ and $2.0 \mathrm{cfu} / \mathrm{g}$ of soil), effective nodules/plant (10.85 and 9.63) and nodules dry weight (81.87 and $73.00 \mathrm{mg} /$ plant) as compare to PGPR, PSB and control during both the years (Tables 1 and 2). Further data showed that higher chlorophyll content (36.0 and 34.55 SPAD), leaf area index (1.58 and 1.26), crop growth rate $(0.436$ and $0.359 \mathrm{~g} /$ day/plant $)$ and dry matter production (21.78 and 17.93) were recorded in PSB + PGPR in both the years, respectively.

Seed inoculation with PSB + PGPR was recorded significantly higher values of yield attributes like pods/plant, seeds/pod, 1000seed weight (Figs. 1, 2 and 3) and seed yield (2314 and $2173 \mathrm{~kg} / \mathrm{ha}$, respectively) of pigeonpea when compared to PGPR, PSB and control in both the years.

Structural and functional characteristics of roots contribute to rhizosphere processes and both have significant influence on the capacity of roots to acquire nutrients.

Roots also interact extensively with soil microorganisms which further impact on plant nutrition either directly, by influencing nutrient availability and uptake, or indirectly through plant (root) growth promotion. These observations are in agreement with those of Singh and Yadav (2008) and Kumawat et al., (2009a) and Singh and Singh (2012b).

Fig.1 Effect of phosphorus and bioinoculants on pods/plant

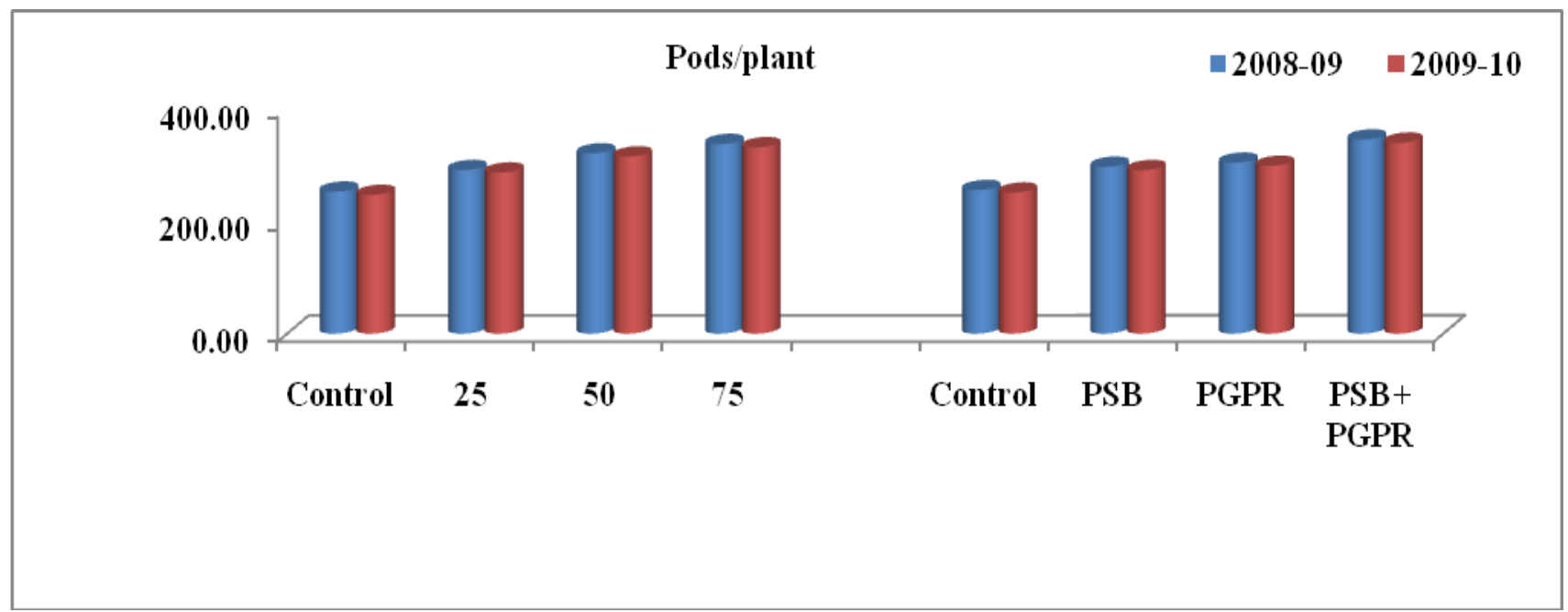


Table.1 Effect of phosphorus levels and bioinoculants on growth attributes of pigeonpea at 50 DAS

\begin{tabular}{|c|c|c|c|c|c|c|c|c|c|c|c|c|c|c|}
\hline \multirow[t]{2}{*}{ Treatment } & \multicolumn{2}{|c|}{$\begin{array}{l}\text { Microbial population } \\
\text { (x } 10^{4} \mathrm{cfu} / \mathrm{g} \text { of soil) }\end{array}$} & \multicolumn{2}{|c|}{$\begin{array}{c}\text { Chlorophyll content } \\
\text { (SPAD) }\end{array}$} & \multicolumn{2}{|c|}{$\begin{array}{c}\text { Effective } \\
\text { nodules/plant }\end{array}$} & \multicolumn{2}{|c|}{$\begin{array}{l}\text { Nodules dry weight } \\
\text { (mg/plant) }\end{array}$} & \multicolumn{2}{|c|}{ Leaf area index (LAI) } & \multicolumn{2}{|c|}{$\begin{array}{c}\text { Crop growth rate } \\
\text { (g/day/plant) }\end{array}$} & \multicolumn{2}{|c|}{ Plant dry weight (g) } \\
\hline & $2008-09$ & $2009-10$ & $2008-09$ & $2009-10$ & $2008-09$ & $2009-10$ & $2008-09$ & $2009-10$ & 2008-09 & $2009-10$ & $2008-09$ & $2009-10$ & $2008-09$ & $2009-10$ \\
\hline \multicolumn{15}{|c|}{ Phosphorus (kg/ha) } \\
\hline Control & 1.1 & 1.5 & 26.63 & 24.48 & 5.95 & 5.43 & 45.08 & 41.10 & 1.31 & 0.99 & 0.323 & 0.249 & 16.13 & 12.43 \\
\hline 25 & 1.3 & 1.7 & 30.45 & 28.83 & 7.33 & 7.01 & 55.52 & 52.96 & 1.44 & 1.11 & 0.362 & 0.286 & 18.09 & 14.29 \\
\hline 50 & 1.4 & 1.9 & 34.28 & 32.33 & 9.45 & 8.69 & 71.63 & 65.78 & 1.57 & 1.24 & 0.415 & 0.341 & 20.75 & 17.03 \\
\hline 75 & 1.5 & 2.0 & 35.50 & 33.75 & 9.93 & 9.16 & 74.41 & 69.35 & 1.57 & 1.25 & 0.429 & 0.353 & 21.43 & 17.64 \\
\hline $\mathrm{SEm} \pm$ & 0.03 & 0.04 & 0.62 & 0.58 & 0.16 & 0.14 & 1.22 & 1.08 & 0.03 & 0.02 & 0.007 & 0.006 & 0.37 & 0.30 \\
\hline $\mathrm{CD}(\mathrm{P}=0.05)$ & 0.09 & 0.12 & 2.13 & 2.00 & 0.56 & 0.50 & 4.20 & 3.75 & 0.10 & 0.08 & 0.026 & 0.021 & 1.29 & 1.03 \\
\hline \multicolumn{15}{|l|}{ Bioinoculants } \\
\hline Control & 1.1 & 1.5 & 28.03 & 25.90 & 6.15 & 5.53 & 46.57 & 41.92 & 1.36 & 1.04 & 0.328 & 0.257 & 16.40 & 12.83 \\
\hline PSB & 1.3 & 1.8 & 31.08 & 29.15 & 7.68 & 7.41 & 58.00 & 56.14 & 1.44 & 1.12 & 0.377 & 0.301 & 18.84 & 15.05 \\
\hline PGPR & 1.4 & 1.9 & 31.75 & 29.78 & 7.98 & 7.71 & 60.21 & 58.13 & 1.50 & 1.18 & 0.388 & 0.312 & 19.38 & 15.58 \\
\hline PSB + PGPR & 1.5 & 2.0 & 36.00 & 34.55 & 10.85 & 9.63 & 81.87 & 73.00 & 1.58 & 1.26 & 0.436 & 0.359 & 21.78 & 17.93 \\
\hline SEm \pm & 0.02 & 0.03 & 0.46 & 0.44 & 0.13 & 0.12 & 0.96 & 0.88 & 0.02 & 0.02 & 0.006 & 0.004 & 0.28 & 0.22 \\
\hline $\mathrm{CD}(\mathrm{P}=0.05)$ & 0.06 & 0.08 & 1.35 & 1.28 & 0.37 & 0.34 & 2.79 & 2.57 & 0.06 & 0.05 & 0.016 & 0.013 & 0.81 & 0.66 \\
\hline
\end{tabular}

Table.2 Effect of phosphorus levels and bioinoculants on growth attributes of pigeonpea at 100 DAS

\begin{tabular}{|c|c|c|c|c|c|c|c|c|c|c|c|c|c|c|}
\hline \multirow[t]{2}{*}{ Treatment } & \multicolumn{2}{|c|}{$\begin{array}{c}\text { Microbial } \\
\text { population (x } 10^{4} \\
\text { cfu/g of soil) }\end{array}$} & \multicolumn{2}{|c|}{$\begin{array}{c}\text { Chlorophyll } \\
\text { content (SPAD) }\end{array}$} & \multicolumn{2}{|c|}{$\begin{array}{c}\text { Effective } \\
\text { nodules/plant }\end{array}$} & \multicolumn{2}{|c|}{$\begin{array}{l}\text { Nodules dry weight } \\
\text { (mg/plant) }\end{array}$} & \multicolumn{2}{|c|}{ Leaf area index (LAI) } & \multicolumn{2}{|c|}{$\begin{array}{l}\text { Crop growth rate } \\
\text { (g/day/plant) }\end{array}$} & \multicolumn{2}{|c|}{$\begin{array}{l}\text { Plant dry weight } \\
\text { (g) }\end{array}$} \\
\hline & 2008-09 & $2009-10$ & 2008-09 & $2009-10$ & 2008-09 & $2009-10$ & 2008-09 & 2009-10 & 2008-09 & $2009-10$ & 2008-09 & $2009-10$ & 2008-09 & $2009-10$ \\
\hline \multicolumn{15}{|c|}{ Phosphorus (kg/ha) } \\
\hline Control & 1.3 & 1.7 & 29.2 & 27.4 & 21.7 & 17.8 & 166.0 & 138.0 & 2.1 & 1.9 & 0.215 & 0.208 & 37.6 & 33.2 \\
\hline 25 & 1.4 & 2.0 & 33.1 & 31.4 & 26.9 & 22.0 & 207.0 & 170.4 & 2.3 & 2.1 & 0.241 & 0.237 & 42.1 & 38.0 \\
\hline 50 & 1.6 & 2.2 & 37.7 & 35.5 & 33.2 & 27.8 & 254.2 & 214.8 & 2.5 & 2.3 & 0.271 & 0.268 & 47.8 & 43.8 \\
\hline 75 & 1.6 & 2.2 & 38.2 & 36.8 & 34.5 & 29.2 & 264.6 & 224.2 & 2.5 & 2.3 & 0.279 & 0.280 & 49.4 & 45.6 \\
\hline SEm \pm & 0.03 & 0.04 & 0.7 & 0.6 & 0.6 & 0.5 & 4.3 & 3.7 & 0.05 & 0.04 & 0.005 & 0.005 & 0.9 & 0.8 \\
\hline $\mathrm{CD}(\mathrm{P}=0.05)$ & 0.10 & 0.14 & 2.3 & 2.2 & 1.9 & 1.7 & 14.8 & 12.7 & 0.16 & 0.14 & 0.017 & 0.017 & 3.0 & 2.7 \\
\hline \multicolumn{15}{|l|}{ Bioinoculants } \\
\hline Control & 1.3 & 1.7 & 30.3 & 28.5 & 23.0 & 18.4 & 178.0 & 142.7 & 2.1 & 1.9 & 0.218 & 0.210 & 38.2 & 33.8 \\
\hline PSB & 1.5 & 2.0 & 34.1 & 32.0 & 28.3 & 22.7 & 218.7 & 176.2 & 2.3 & 2.1 & 0.249 & 0.246 & 43.7 & 39.63 \\
\hline PGPR & 1.6 & 2.1 & 35.4 & 33.1 & 29.9 & 23.6 & 223.5 & 181.9 & 2.4 & 2.2 & 0.255 & 0.253 & 44.9 & 40.8 \\
\hline PSB + PGPR & 1.7 & 2.3 & 38.4 & 37.5 & 35.1 & 32.2 & 271.6 & 246.7 & 2.5 & 2.3 & 0.283 & 0.284 & 50.1 & 46.3 \\
\hline SEm \pm & 0.02 & 0.03 & 0.5 & 0.4 & 0.5 & 0.4 & 3.3 & 2.9 & 0.03 & 0.03 & 0.004 & 0.004 & 0.6 & 0.6 \\
\hline $\mathrm{CD}(\mathrm{P}=0.05)$ & 0.06 & 0.08 & 1.4 & 1.3 & 1.3 & 1.1 & 9.8 & 8.4 & 0.10 & 0.09 & 0.011 & 0.011 & 1.9 & 1.7 \\
\hline
\end{tabular}


Table.3 Effect of phosphorus levels and bioinoculants on protein content $(\%)$, yield ( $\mathrm{kg} / \mathrm{ha})$ and economics of pigeonpea

\begin{tabular}{|c|c|c|c|c|c|c|c|c|c|c|c|c|c|c|}
\hline \multirow{3}{*}{ Treatment } & \multicolumn{4}{|c|}{ Protein } & \multirow{2}{*}{\multicolumn{2}{|c|}{$\begin{array}{c}\text { Seed } \\
\text { yield } \\
(\mathrm{kg} / \mathrm{ha})\end{array}$}} & \multirow{2}{*}{\multicolumn{2}{|c|}{$\begin{array}{c}\text { Net } \\
\text { return } \\
\left(\times 10^{3} / \mathrm{ha}\right)\end{array}$}} & \multirow{2}{*}{\multicolumn{2}{|c|}{$\begin{array}{l}\text { B: C } \\
\text { ratio }\end{array}$}} & \multicolumn{4}{|c|}{ Efficiency } \\
\hline & \multicolumn{2}{|c|}{$\begin{array}{c}\text { Content } \\
(\%)\end{array}$} & \multicolumn{2}{|c|}{$\begin{array}{c}\text { Yield } \\
\text { (kg/ha) }\end{array}$} & & & & & & & \multicolumn{2}{|c|}{$\begin{array}{l}\text { Production } \\
\text { (kg/ha/day) }\end{array}$} & \multicolumn{2}{|c|}{$\begin{array}{l}\text { Economic } \\
\text { ('/ha/day) }\end{array}$} \\
\hline & 2008-09 & $2009-10$ & $2008-09$ & $2009-10$ & $2008-09$ & $2009-10$ & 2008-09 & $2009-10$ & $2008-09$ & $2009-10$ & $2008-09$ & $2009-10$ & 2008-09 & $2009-10$ \\
\hline \multicolumn{15}{|c|}{ Phosphorus (kg/ha) } \\
\hline Control & 19.15 & 18.36 & 321.4 & 277.7 & 1673 & 1508 & 64.27 & 56.87 & 2.30 & 2.03 & 6.3 & 5.6 & 240.7 & 212.9 \\
\hline 25 & 19.55 & 19.01 & 380.9 & 342.1 & 1945 & 1795 & 78.23 & 71.25 & 2.75 & 2.51 & 7.3 & 6.7 & 292.9 & 266.8 \\
\hline 50 & 19.70 & 19.26 & 437.4 & 399.2 & 2218 & 2068 & 91.76 & 85.50 & 3.18 & 2.96 & 8.3 & 7.7 & 343.7 & 320.2 \\
\hline 75 & 19.97 & 19.52 & 452.5 & 415.3 & 2265 & 2124 & 93.43 & 87.66 & 3.19 & 2.99 & 8.5 & 7.9 & 349.9 & 328.3 \\
\hline SEm \pm & 0.39 & 0.38 & 7.6 & 6.7 & 39 & 36 & 1.55 & 1.41 & 0.05 & 0.05 & 0.14 & 0.13 & 5.8 & 5.3 \\
\hline $\mathrm{CD}(\mathrm{P}=0.05)$ & NS & NS & 26.4 & 23.4 & 134 & 123 & 5.36 & 4.89 & 0.19 & 0.17 & 0.50 & 0.46 & 20.1 & 18.3 \\
\hline \multicolumn{15}{|l|}{ Bioinoculants } \\
\hline Control & 19.15 & 18.47 & 325.2 & 283.7 & 1693 & 1530 & 65.37 & 57.57 & 2.29 & 2.02 & 6.3 & 5.7 & 244.8 & 215.6 \\
\hline PSB & 19.54 & 18.94 & 391.1 & 350.5 & 1999 & 1846 & 80.46 & 74.31 & 2.81 & 2.59 & 7.5 & 6.9 & 301.4 & 278.3 \\
\hline PGPR & 19.64 & 19.21 & 412.2 & 374.6 & 2095 & 1945 & 85.17 & 79.09 & 2.97 & 2.76 & 7.8 & 7.3 & 318.9 & 296.2 \\
\hline PSB + PGPR & 20.04 & 19.53 & 463.8 & 425.6 & 2314 & 2173 & 96.70 & 90.30 & 3.34 & 3.12 & 8.7 & 8.1 & 362.2 & 338.2 \\
\hline $\mathrm{SEm} \pm$ & 0.27 & 0.26 & 5.8 & 5.3 & 29 & 27 & 1.21 & 1.12 & 0.04 & 0.04 & 0.11 & 0.10 & 4.5 & 4.2 \\
\hline $\mathrm{CD}(\mathrm{P}=0.05)$ & NS & NS & 17.0 & 15.5 & 86 & 80 & 3.53 & 3.27 & 0.12 & 0.11 & 0.32 & 0.30 & 13.2 & 12.3 \\
\hline
\end{tabular}

Table.4 Correlation coefficients showing relationship between independent variables (yield attributes)

And dependent variable (seed yield) of pigeonpea

\begin{tabular}{|c|c|c|c|c|c|c|c|c|c|c|c|c|}
\hline \multirow{2}{*}{ Character } & \multicolumn{2}{|c|}{$\begin{array}{l}\text { Pod length } \\
(\mathrm{cm})\end{array}$} & \multicolumn{2}{|c|}{$\begin{array}{l}\text { Pods/plant } \\
\text { (Number) }\end{array}$} & \multicolumn{2}{|c|}{$\begin{array}{l}\text { Pods weight/plant } \\
(\mathrm{g})\end{array}$} & \multicolumn{2}{|c|}{$\begin{array}{l}\text { Seeds/pod } \\
\text { (Number) }\end{array}$} & \multicolumn{2}{|c|}{$\begin{array}{c}\text { 1000-seed weight } \\
(\mathrm{g})\end{array}$} & \multicolumn{2}{|c|}{$\begin{array}{l}\text { Seed yield } \\
(\mathrm{kg} / \mathrm{ha})\end{array}$} \\
\hline & 2008-09 & $2009-10$ & 2008-09 & $2009-10$ & 2008-09 & $2009-10$ & 2008-09 & $2009-10$ & 2008-09 & $2009-10$ & $\begin{array}{c}2008- \\
09\end{array}$ & $\begin{array}{c}2009- \\
10\end{array}$ \\
\hline Pod length $(\mathrm{cm})$ & 1.00 & 1.00 & & & & & & & & & & \\
\hline Pods/plant & $0.988 * *$ & $0.993 * *$ & 1.00 & 1.00 & & & & & & & & \\
\hline $\begin{array}{l}\text { Pods weight/plant } \\
\text { (g) }\end{array}$ & $0.993 * *$ & $0.992 * *$ & $0.985^{* *}$ & $0.987 * *$ & 1.00 & 1.00 & & & & & & \\
\hline Seeds/pod & $0.992 * *$ & $0.985 * *$ & $0.988 * *$ & $0.990 * *$ & $0.991 * *$ & $0.992 * *$ & 1.00 & 1.00 & & & & \\
\hline $\begin{array}{l}\text { 1000-seed weight } \\
\text { (g) }\end{array}$ & $0.988 * *$ & $0.985 * *$ & $0.980 * *$ & $0.981 * *$ & $0.992 * *$ & $0.994 * *$ & $0.984 * *$ & $0.982 * *$ & 1.00 & 1.00 & & \\
\hline Seed yield $(\mathrm{kg} / \mathrm{ha})$ & $0.984 * *$ & $0.985 * *$ & $0.979 * *$ & $0.983 * *$ & $0.990 * *$ & $0.991 * *$ & $0.993 * *$ & $0.992 * *$ & $0.985 * *$ & $0.985 * *$ & 1.00 & 1.00 \\
\hline
\end{tabular}

** Significant at $1 \%$ level of significance 
Table.5 Effect of phosphorus levels and bioinoculants on organic carbon (\%) and available soil nutrient (kg/ha)

\begin{tabular}{|c|c|c|c|c|c|c|c|c|c|c|}
\hline \multirow{3}{*}{ Treatment } & \multirow{2}{*}{\multicolumn{2}{|c|}{ Organic carbon $(\%)$}} & \multicolumn{8}{|c|}{ Available soil nutrient (kg/ha) } \\
\hline & & & \multicolumn{2}{|c|}{ Nitrogen } & \multicolumn{2}{|c|}{ Phosphorus } & \multicolumn{2}{|c|}{ Potassium } & \multicolumn{2}{|c|}{ Sulphur } \\
\hline & $2008-09$ & $2009-10$ & $2008-09$ & $2009-10$ & $2008-09$ & $2009-10$ & $2008-09$ & $2009-10$ & $2008-09$ & $2009-10$ \\
\hline \multicolumn{11}{|c|}{ Phosphorus (kg/ha) } \\
\hline Control & 0.24 & 0.26 & 188.3 & 183.5 & 15.5 & 14.3 & 206.7 & 204.5 & 14.2 & 13.2 \\
\hline 25 & 0.31 & 0.33 & 202.9 & 213.3 & 17.7 & 21.4 & 215.2 & 222.5 & 16.6 & 18.7 \\
\hline 50 & 0.39 & 0.41 & 217.0 & 227.3 & 19.8 & 23.5 & 226.5 & 233.2 & 18.5 & 21.0 \\
\hline 75 & 0.41 & 0.43 & 217.7 & 228.0 & 20.3 & 24.0 & 227.1 & 234.2 & 19.2 & 21.9 \\
\hline SEm \pm & 0.006 & 0.007 & 4.0 & 4.1 & 0.4 & 0.4 & 4.4 & 4.4 & 0.3 & 0.3 \\
\hline $\mathrm{CD}(\mathrm{P}=0.05)$ & 0.022 & 0.023 & 14.0 & 14.1 & 1.2 & 1.3 & 15.0 & 15.3 & 1.1 & 1.2 \\
\hline \multicolumn{11}{|l|}{ Bioinoculants } \\
\hline Control & 0.27 & 0.29 & 194.2 & 200.7 & 15.5 & 17.8 & 210.3 & 214.3 & 14.2 & 15.2 \\
\hline PSB & 0.32 & 0.35 & 205.4 & 211.9 & 17.7 & 20.4 & 218.0 & 219.6 & 16.6 & 17.9 \\
\hline PGPR & 0.34 & 0.36 & 208.5 & 215.0 & 19.3 & 21.8 & 219.8 & 225.3 & 18.2 & 20.0 \\
\hline PSB + PGPR & 0.42 & 0.44 & 217.8 & 224.4 & 20.7 & 23.2 & 227.2 & 235.2 & 19.6 & 21.7 \\
\hline $\mathrm{SEm} \pm$ & 0.004 & 0.005 & 2.9 & 3.0 & 0.3 & 0.3 & 3.1 & 3.2 & 0.3 & 0.3 \\
\hline $\mathrm{CD}(\mathrm{P}=0.05)$ & 0.015 & 0.016 & 8.4 & 8.8 & 0.8 & 0.9 & 8.9 & 9.2 & 0.7 & 0.8 \\
\hline
\end{tabular}

Table.6 Linear regression coefficients equations showing relationship between independent variables (Pods/plant, seeds/pod and 1000-seed weight) and dependent variable (seed yield) of pigeonpea

\begin{tabular}{|c|c|c|c|}
\hline \multirow[t]{2}{*}{$\begin{array}{c}\text { Dependent variables } \\
\text { (Y) }\end{array}$} & \multirow[t]{2}{*}{ Independent variables $(\mathrm{X})$} & \multicolumn{2}{|c|}{$\begin{array}{l}\text { Regression equations } \\
\qquad \mathrm{Y}=\mathrm{a}+\mathrm{b}_{\mathrm{yx}} \mathrm{X}\end{array}$} \\
\hline & & 2008-09 & $2009-10$ \\
\hline \multirow[t]{3}{*}{ Seed yield (kg/ha) } & $\mathrm{X}_{1}$ Pods/plant & $\mathrm{Y}=-0.867+0.070 \mathrm{X}_{1}$ & $Y=-2.922+0.073 X_{1}$ \\
\hline & $\mathrm{X}_{2}$ Seeds/pod & $Y=-1.302+5.409 X_{2}$ & $\mathrm{Y}=-2.311+5.415 \mathrm{X}_{2}$ \\
\hline & $\mathrm{X}_{3} 1000$-seed weight $(\mathrm{g})$ & $Y=0.605+0.180 X_{3}$ & $Y=-0.067+0.183 X_{3}$ \\
\hline
\end{tabular}


Fig.2 Effect of phosphorus and bioinoculants on seeds/pod

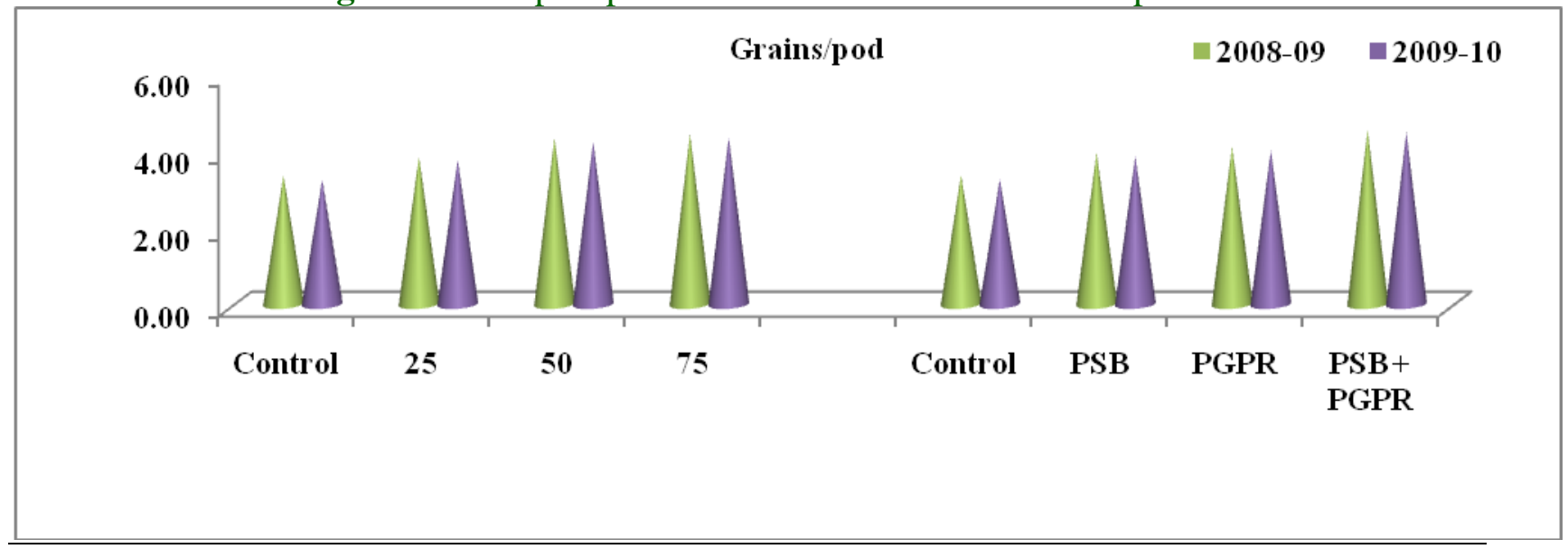

Fig.3 Effect of phosphorus and bioinoculants on 1000-seed weight (g)

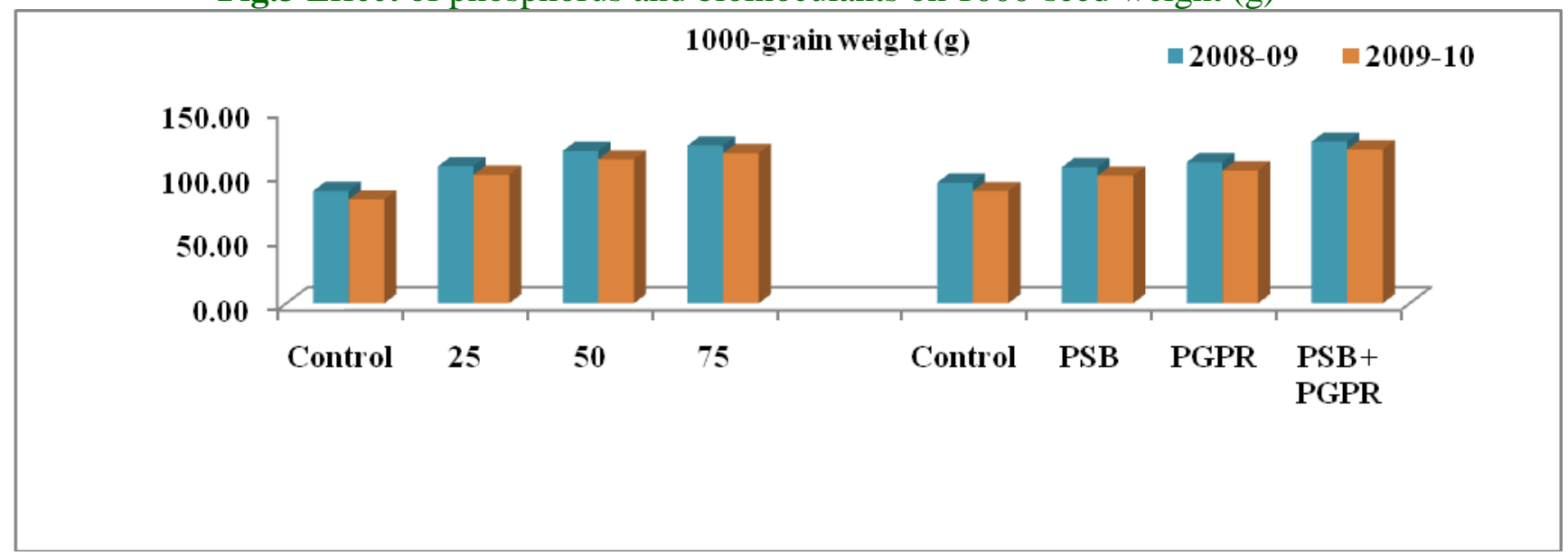

Further table 3 shows that dual inoculation of PSB + PGPR gave maximum net returns of 96.70 and $90.30\left(\times 10^{3} / \mathrm{ha}\right)$, benefit: cost ratio of 3.34 and 3.12, production efficiency of 8.7 and $8.1(\mathrm{~kg} / \mathrm{ha} / \mathrm{day})$ and economic efficiency of 362.2 and 338.2 ( $/$ ha/day), it being significantly superior to seed inoculation alone with PGPR, PSB and control, respectively (Table 3). Among the bioinoculants, dual seed inoculation with PSB + PGPR was recorded higher protein content which was significantly superior to rest of the bioinoculants during both the years (Table 3 ). Similarly, the highest protein yield was noted with seed inoculation with PSB + PGPR, being significantly superior to PGPR, PSB alone and control. This might be due to direct enhancement of nutrient uptake $(\mathrm{N})$ due to increases in specific ion fluxes at the root surface in the presence of PGPR. Similar results were also reported by Singh and Yadav (2008), Gupta et al., (2009) and Singh et al., (2012). The higher amount of available organic carbon was recorded with seed inoculation of PSB + PGPR and this treatment was significantly superior to seed inoculation with PSB and control. Seed inoculation of PSB + PGPR gave maximum available soil N, $\mathrm{P}, \mathrm{K}$ and sulphur in soil after harvest of crop but it was statistically similar with seed inoculation of PGPR and significantly superior to seed inoculation of PSB and control (Table 5). It might be due to release of the native $\mathrm{P}$ from the soil and increased concentration of phosphorus in soil solution which helped in early root growth and 
nodules formation. As a result, symbiotic $\mathrm{N}_{2-}$ fixation increased legumes. Due to increase in symbiotic $\mathrm{N}_{2}$-fixation, available soil nutrients in soil also increased. These observations are in agreement with those of Singh and Singh (2012b).

\section{Correlation and regression coefficient}

Correlation between yield attributes (pod length, pods/plant, pod weight/plant, seeds/pod and 1000-seed weight) and seed yield was observed (Table 4). The correlation coefficient between yields attributes and seed yield showed that it was highly correlated with each other. From the regression equation of pods/plant, seeds/pod and 1000-seed weight (Table 6).

It was concluded that to maximize the productivity, profitability; quality of pigeonpea along with sustained soil fertility, the crop pigeonpea may be fertilized with 75 $\mathrm{kg} \mathrm{P}_{2} \mathrm{O}_{5} /$ ha along with seed inoculation of PSB + PGPR under irrigated ecosystem of Varanasi in Eastern Uttar Pradesh.

\section{Acknowledgement}

The authors acknowledge the Head, Department of Agronomy for providing necessary facilities for conducting experiment and esteemed referee for revision of manuscript whose comments and advice has greatly enhanced this article. The first author also thanks to the UGC, New Delhi for providing fellowship during research programme.

\section{References}

Ahirwar, R.P., Mishra, U.S., Mitra, N.G., Siothia, P. and Ahirwar, K. 2016. Effect of phosphorus and bio-fertilizers on nutrient contents and uptake by pigeon pea (Cajanus cajan L.) and residual soil constituents. International Journal of Agriculture Sciences, 8 (54): 29432945.

Ali, M. and Gupta, S. 2012. Carrying capacity of Indian agriculture: pulse crops. Current Science, 102 (6): 874-881.

Chandra, R. and Kumar, S. 2005. Biofertilizers: An eco-friendly source of plant nutrient. Indian Farmers Digest, 4: 8-9.

Gupta, S.C., Singh, R.P. and Verma, R. 2009. Response of chickpea (Cicer arietinum L.) to phosphorus levels from different sources with various PSB species. Legume Research, 32 (3): 212-214.

Jain, A.K., Kumar, S. and Panwar, J.D.S. 2007. Response of mungbean (Vigna radiata) to phosphorus and micronutrients on $\mathrm{N}$ and $\mathrm{P}$ uptake and seed quality. Legume Research, 30 (3): 201-204.

Kumar, A. and Kushwaha, H.S. 2006. Response of pigeonpea (Cajanus cajan) to sources and levels of phosphorus under rainfed condition. Indian Journal of Agronomy, 51 (1): 60-62.

Kumawat, N., Sharma, O.P. and Kumar, R. 2009a Nutrient uptake and yield of mungbean (Vigna radiata L.) as influenced by organic manure, PSB and $\mathrm{P}$ fertilization. Environment and Ecology, 27: 2002-2005.

Kumawat, N., Sharma, O.P., Kumar, R. 2009b. Effect of organic manure, PSB and phosphorus fertilization on yield and economics of mungbean (Vigna radiata). Environment and Ecology, 27: 5-7.

Kumawat, N., Sharma, O.P., Kumar, R., Kumari, A. 2010. Yield and yield attributes of mungbean [Vigna radiata (L.) Wilczek] as affected by organic manures, PSB and phosphorus fertilization. Environment and Ecology, 28 (1A):332-335.

Kumawat, N., Sharma, O.P., Kumar, R. and 
Kumari, A. 2009c. Response of organic manures, PSB and phosphorus fertilization on growth and yield of mungbean. Environment and Ecology, 27 (4B): 2024-2027.

Kumawat, A., Pareek, B.L. and Yadav, R.S. 2010. Response of greengram (Vigna radiata) to biofertilizers under different fertility levels. Indian Journal of Agricultural Sciences, 80 (7): 655-657.

Kumawat, N., Singh, R.P., Kumar, R. and Hari, Om 2013. Effect of integrated nutrient management on the performance of sole and intercropped pigeonpea (Cajanus cajan) under rainfed conditions. Indian J. Agron. 58: 309-315.

Kumawat, N., Singh, R.P., Kumar, R., Kumari, A. and Kumar, P. 2012. Response of intercropping and integrated nutrition on production potential and profitability on rainfed pigeonpea. J. Agric Sci. 4: 154-162.

Kumawat, N., Singh, R.P., Kumar, R., Yadav, T.P. and Hari, Om 2015. Effect of integrated nutrient management on productivity, nutrient uptake and economics of rainfed pigeonpea (Cajanus cajan) and blackgram (Vigna mungo) intercropping system. Indian J. Agric. Sci. 85: 171-176.

Podile, A.R. and Kishore, G.K. 2006. Plant growth promoting rhizobacteria. S.S.
Gnanamanickam (Ed.), PlantAssociated Bacteria, pp. 195-230.

Singh, A.K. and Singh, R.S. 2012b. Effect of phosphorus and bioinoculants on yield, nutrient uptake and economics of long duration pigeonpea (Cajanus cajan). Indian Journal of Agronomy, 57 (3): 265-269.

Singh, A.K. and Singh, R.S. 2012a. Effect phosphorus levels and bioinoculants on growth and yield of long duration pigeonpea [Cajanus cajan (L.) Millsp.]. Journal of Food Legumes, 25 (1): 7375.

Singh, R.S. and Yadav, M.K. 2008. Effect of phosphorus and biofertilizers on growth, yield and nutrient uptake of long duration pigeonpea under rainfed condition. Journal of Food Legume, 21 (1): 46-48.

Singh, S.R., Najar, G.R. and Singh, U. 2012. Phosphorus management in field pea (Pisum sativum)-rice (Oryza sativa) cropping system under temperate conditions. Indian Journal of Agricultural Sciences, 82 (6): 494-499.

Singh, U. and Ahlawat, I.P.S. 2007. Phosphorus management in pigeonpea (Cajanus cajan L.)-wheat (Triticum aestivum L.) cropping system. Indian Journal of Agronomy, 52 (1): 21-26.

\section{How to cite this article:}

Amitesh Kumar Singh, R.S. Singh, S.P. Singh, Narendra Kumawat and Rakesh Kumar. 2017. Productivity, profitability and soil health of pigeonpea as influenced by phosphorus levels and bioinoculants under eastern Uttar Pradesh. Int.J.Curr.Microbiol.App.Sci. 6(6): 1723-1732. doi: https://doi.org/10.20546/ijcmas.2017.606.200 